\title{
Histone 2A Family Member J Drives Mesenchymal Transition and Temozolomide Resistance in Glioblastoma Multiforme
}

\author{
Hsun-Hua Lee ${ }^{1,2,3,4,5}$ (D), Che-Hsuan Lin ${ }^{6,7,8}$, Hui-Yu Lin ${ }^{1,9}{ }^{(D}$, Chia-Hao Kuei 1,10, \\ Jing-Quan Zheng 1,11, Yuan-Hung Wang 1,12, Long-Sheng Lu 13,14, Fei-Peng Lee 8,15, \\ Chaur-Jong $\mathrm{Hu}^{1,2}$, Dean $\mathrm{Wu}^{1,2,4,5,16, * \mathbb{D}}$ and Yuan-Feng Lin 1,17,*(D)
}

1 Graduate Institute of Clinical Medicine, College of Medicine, Taipei Medical University, Taipei 11031, Taiwan; kaorulei@yahoo.com.tw (H.-H.L.); candycaro10227@gmail.com (H.-Y.L.); pplay1028@gmail.com (C.-H.K.); 16044@s.tmu.edu.tw (J.-Q.Z.); d508091002@tmu.edu.tw (Y.-H.W.); chaurjongh@tmu.edu.tw (C.-J.H.)

2 Department of Neurology, School of Medicine, College of Medicine, Taipei Medical University, Taipei 11031, Taiwan

3 Dizziness and Balance Disorder Center, Shuang-Ho Hospital, Taipei Medical University, New Taipei City 23561, Taiwan

4 Department of Neurology, Shuang-Ho Hospital, Taipei Medical University, New Taipei City 23561, Taiwan

5 Taipei Neuroscience Institute, Taipei Medical University, New Taipei City 23561, Taiwan

6 Graduate Institute of Medical Sciences, College of Medicine, Taipei Medical University, Taipei 11031, Taiwan; cloudfrank@gmail.com

7 Department of Otolaryngology, Taipei Medical University Hospital, Taipei Medical University, Taipei 11031, Taiwan

8 Department of Otolaryngology, School of Medicine, College of Medicine, Taipei Medical University, Taipei 11031, Taiwan; fplee@tmu.edu.tw

9 Breast Center, Department of General Surgery, Cardinal Tien Hospital, Xindian District, New Taipei City 231, Taiwan

10 Urology, Division of Surgery, Cardinal Tien Hospital, Xindian District, New Taipei City 231, Taiwan

11 Department of Critical Care Medicine, Shuang Ho Hospital, Taipei Medical University, New Taipei City 23561, Taiwan

12 Department of Medical Research, Shuang-Ho Hospital, Taipei Medical University, New Taipei City 23561, Taiwan

13 Department of Radiation Oncology, Taipei Medical University Hospital, Taipei Medical University, Taipei 11031, Taiwan; lslu@tmu.edu.tw

14 Graduate Institute of Biomedical Materials and Tissue Engineering, College of Biomedical Engineering, Taipei Medical University, Taipei 11031, Taiwan

15 Department of Otolaryngology, Shuang-Ho Hospital, Taipei Medical University, New Taipei City 23561, Taiwan

16 Sleep Center, Shuang-Ho Hospital, Taipei Medical University, New Taipei City 23561, Taiwan

17 Cell Physiology and Molecular Image Research Center, Wan Fang Hospital, Taipei Medical University, Taipei 116, Taiwan

* Correspondence: d102095020@tmu.edu.tw (D.W.); d001089012@tmu.edu.tw (Y.-F.L.); Tel.: +886-2-22490088 (ext. 8112) (D.W.); +886-2-2736-1661 (ext. 3106) (Y.-F.L.); Fax: +886-2-22490088 (D.W.); +886-2-2739-0500 (Y.-F.L.)

Received: 29 November 2019; Accepted: 27 December 2019; Published: 30 December 2019

\begin{abstract}
Glioblastoma multiforme (GBM) is the most aggressive brain tumor and has a poor prognosis and is poorly sensitive to radiotherapy or temozolomide (TMZ) chemotherapy. Therefore, identifying new biomarkers to predict therapeutic responses of GBM is urgently needed. By using The Cancer Genome Atlas (TCGA) database, we found that the upregulation of histone 2A family member $\mathrm{J}(\mathrm{H} 2 \mathrm{AFJ})$, but not other H2AFs, is extensively detected in the therapeutic-insensitive mesenchymal, IDH wildtype, MGMT unmethylated, or non-G-CIMP GBM and is associated with poor TMZ
\end{abstract}


responsiveness independent of radiation. Similar views were also found in GBM cell lines. Whereas $\mathrm{H} 2 \mathrm{AFJ}$ knockdown diminished TMZ resistance, H2AFJ overexpression promoted TMZ resistance in a panel of GBM cell lines. Gene set enrichment analysis (GSEA) revealed that H2AFJ upregulation accompanied by the activation of TNF- $\alpha /$ NF- $\mathrm{BB}$ and IL-6/STAT3-related pathways is highly predicted. Luciferase-based promoter activity assay further validated that the activities of NF- $\mathrm{KB}$ and STAT3 are causally affected by H2AFJ expression in GBM cells. Moreover, we found that therapeutic targeting HADC3 by tacedinaline or NF- $\mathrm{KB}$ by ML029 is likely able to overcome the TMZ resistance in GBM cells with H2AFJ upregulation. Significantly, the GBM cohorts harboring a high-level H2AFJ transcript combined with high-level expression of TNF- $\alpha / N F-\kappa B$ geneset, IL-6/STAT3 geneset or HADC3 were associated with a shorter time to tumor repopulation after initial treatment with TMZ. These findings not only provide $\mathrm{H} 2 \mathrm{AFJ}$ as a biomarker to predict TMZ therapeutic effectiveness but also suggest a new strategy to combat TMZ-insensitive GBM by targeting the interaction network constructed by TNF- $\alpha /$ NF-kB, IL-6/STAT3, HDAC3, and H2AFJ.

Keywords: GBM; Temozolomide; H2AFJ; MGMT; precision medicine

\section{Introduction}

Glioblastoma multiforme (GBM) is the most aggressive brain tumor and is a grade IV histological malignancy, according to the WHO classification [1]. Patients with GBM have a poor prognosis, reported as only a $4-5 \%$ five-year survival rate [2,3] and a median survival time of approximately $14-15$ months from diagnosis, probably owing to the poor response to radiotherapy and chemotherapy $[4,5]$. Based on molecular signatures, GBM has been recently classified into proneural, neural, classical, and mesenchymal subtypes [6]. In comparison with other subtypes, mesenchymal-type GBM has been shown to be strongly associated with unfavorable outcomes, e.g., temozolomide (TMZ) resistance, in clinical patients. Since TMZ is a major chemotherapy drug used to treat GBM patients, uncovering the molecular mechanism underlying TMZ resistance is urgently needed.

Recent studies have demonstrated that $12 \%$ of GBM patients, especially young patients and patients with secondary GBM, acquire mutations in the active site of isocitrate dehydrogenase 1 (IDH1) $[7,8]$. IDH1 plays a substantial role in relieving cellular oxidative damage through the enhancement of NADPH production [7]. Prognostic estimations have revealed that an IDH1 mutation is associated with an increased survival probability in glioma patients who receive TMZ treatment $[9,10]$. In addition, the upregulation of $\mathrm{O}^{6}$-methylguanine DNA methyltransferase (MGMT), a DNA repair enzyme, has been associated with a poor response to TMZ treatment in GBM patients [11,12]. As a result, the hypermethylation of the GC island in the MGMT promoter has been used as a predictive marker for a prolonged survival time in GBM patients undergoing TMZ therapy [13]. Therefore, identifying more powerful biomarkers for predicting TMZ therapeutic effectiveness is worthwhile to more precisely estimate the outcomes of GBM patients who are deciding whether to receive TMZ treatment.

Histone 2A family members (H2AFs) are one of the components of nucleosomes and are crucial for gene regulation in the host [14]. Physiologically, H2AFX has been shown to promote the maintenance of genome integrity in male germ cells [15]. In breast cancer, copy number alterations and promoter genetic variations appear to correlate with breast carcinogenesis and the risk of sporadic breast cancer [16]. Accordingly, the genetic variation of the H2AFX promoter has been associated with the risk of glioma, particularly adult glioma [17]. On the other hand, the fusion of H2AFY with MDS1 and EVI1 Complex (MECOM) and the downregulation of H2AFZ have been associated with cancer progression in leukemia $[18,19]$. However, the clinical relevance of H2AFJ in cancers, including GBM, remains unknown.

Here, we show that H2AFJ is upregulated in primary tumors, compared to its expression in normal tissues derived from GBM patients. Moreover, our results indicate that the upregulation of 
H2AFJ, but not other H2AFs, is commonly found in mesenchymal-type GBM and is significantly associated with poor overall survival rates, probably due to the poor response to TMZ treatment in GBM patients with an unmethylated MGMT promoter region. Artificially silencing H2AFJ enhanced TMZ cytotoxicity against GBM cells, whereas overexpressing exogenous H2AFJ rendered GBM cells more resistant to TMZ treatment. Furthermore, we found that H2AFJ upregulation may be associated with the proneural-mesenchymal transition, which correlates with TMZ resistance [20] and likely activates TNF- $\alpha / \mathrm{NF}-\kappa \mathrm{B}$ pathway which has been shown to mediate mesenchymal differentiation and therapeutic resistance in GBM cells [21]. Significantly, our results revealed that the therapeutic targeting of class I histone deacetylases (HDACs), e.g., HDAC3, by tacedinaline, which is a phase II clinical trial agent against advanced pancreatic cancer [22], might be a new strategy to combat TMZ-resistant GBM with H2AFJ upregulation.

\section{Results}

2.1. H2AFJ Is Frequently Upregulated in Mesenchymal-Type GBM Compared to Normal Brain Tissues and Low-Grade Gliomas

We first analyzed the transcriptional profile of these genes analyzed by microarray method using Agilent_2 platform in TCGA normal brain tissues and GBM subtypes (pro-neural, neural, classical and mesenchymal) (Figure 1A). The results demonstrated that the mRNA levels of H2AFJ, H2AFV, H2AFX, $H 2 A F Y$, and H2AFZ were upregulated in primary tumors compared to the levels in normal brain tissues derived from GBM patients (Figure 1B). Intriguingly, transcriptional profiling revealed that the gene expression of $\mathrm{H} 2 \mathrm{AFJ}$, but not other $\mathrm{H} 2 \mathrm{~A}$ histone family members (H2As), was significantly $(p<0.005)$ upregulated in mesenchymal-type GBM tissues but relatively lower in proneural-type GBM tissues (Figure 1A,B). In contrast, the transcripts of $H 2 A F V, H 2 A F X, H 2 A F Y$, and $H 2 A F Z$ were poorly expressed in mesenchymal-type GBM tissues but highly expressed in proneural-type GBM tissues (Figure 1A,B). Similar views were also observed in the dissection of their mRNA levels analyzed by RNA sequencing technique in TCGA normal brain tissues and GBM subtypes (Figure S1A,B). Kaplan-Meier analyses demonstrated that H2AFJ, but not other H2As, at higher mRNA levels determined by the median of its transcription profiling using Agilent microarray in TCGA GBM tissues significantly $(p=0.016)$ predict a poor overall survival probability (Figure 1C). Based on these findings, we thereafter focused on investigating the clinical relevance of $\mathrm{H} 2 \mathrm{AFJ}$ in GBM.

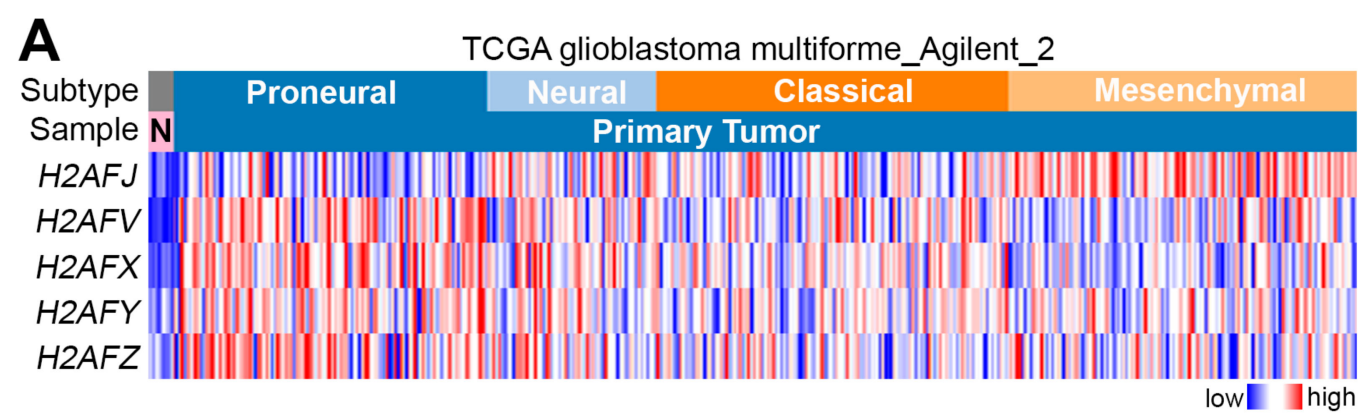

Figure 1. Cont. 


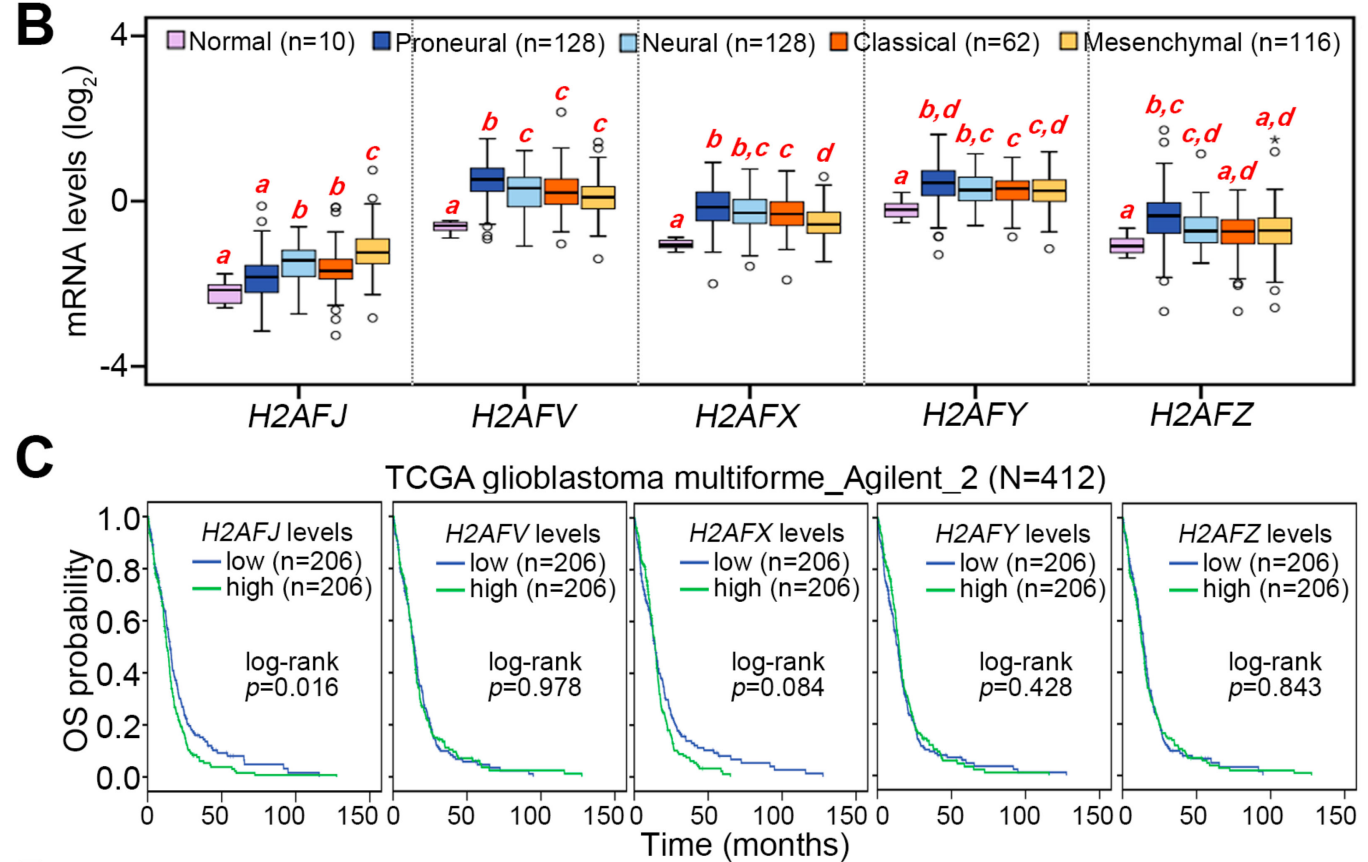

D

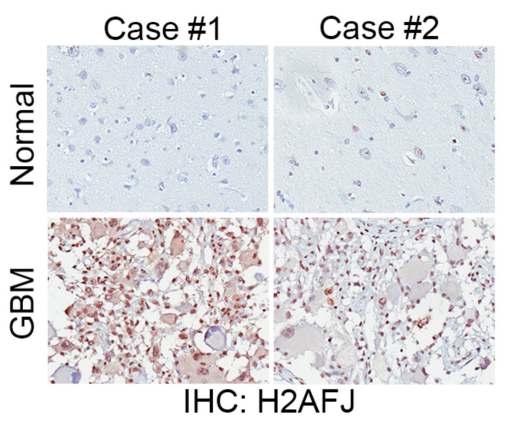

\section{E}

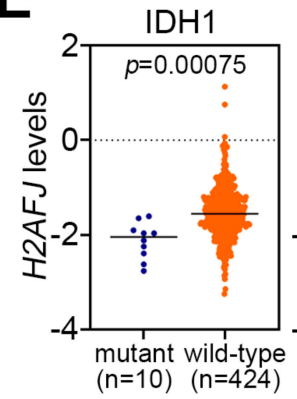

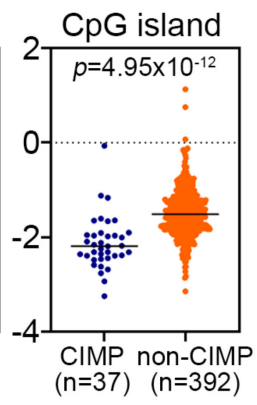

Figure 1. H2AFJ is highly expressed in mesenchymal-type GBM tissues. (A,B) Heatmap (A) and boxplot (B) for the transcriptional profile of the H2A subfamily, which was analyzed by Agilent G4502A microarray, in normal brain tissues ( $\mathrm{N}$ for heatmap) and primary tumors derived from patients with different molecular subtypes (proneural, neural, classical and mesenchymal) of GBM using TCGA database. In (B), statistical significance was estimated by one-way ANOVA and Turkey's post-hoc test. (C) Kaplan-Meier analyses for the mRNA levels of H2A subfamily under the condition of overall survival (OS) probability using TCGA GBM database. (D) Immunohistochemistry (IHC) staining of H2AFJ protein in two representatives of normal brain and GBM tissues. Photographs were taken at a magnification of $400 \times$. (E) Dot plots for the transcriptional profiling of H2AFJ in IDH1 mutant and wild-type GBM, MGMT promoter methylated (Me), and unmethylated (Ume) GBM, or CpG island methylation phenotype (CIMP) and non-CIMP-harboring GBM. The statistical significance was determined by Student's t-test.

Similar to the transcriptional levels, H2AFJ protein expression examined by immunohistochemistry staining was dramatically upregulated in GBM compared to normal brain tissues (Figure 1D) even though the sample size was not sufficient. Since IDH1 mutation, MGMT promoter methylation, and CpG island methylation phenotype (CIMP) have been widely used to estimate the effectiveness of radiation and TMZ therapies on GBM patients, we next analyzed the transcription profiling of $\mathrm{H} 2 \mathrm{AFJ}$ in these molecular classifications. Whereas the mRNA levels of other H2As were not robustly different (Figure S2A-C), the mRNA levels of H2AFJ were significantly $(p<0.001)$ higher in GBM with wild-type IDH1, MGMT promoter unmethylation or non-CIMP, respectively, than in GBM with IDH1 mutations, MGMT promoter methylation or CIMP (Figure 1E). It suggests that H2AFJ upregulation might be associated with a poor radiation or TMZ response in GBM patients. 


\subsection{H2AFJ Upregulation Is Highly Correlated with a Poor Prognosis in Patients with Brain Tumors}

To understand the clinical relevance of $H 2 A F J$, we next performed a meta-analysis using the PrognoScan database (http://www.prognoscan.org/). Our data showed that H2AFJ upregulation was associated with unfavorable hazard ratios in different types of cancer, especially in brain tumors (Figure 2A). We further validated these findings by performing a Kaplan-Meier analysis for patients with low-grade glioma (LGG) and/or GBM from TCGA using the SurvExpress program [23]. The data showed that an elevated level of H2AFJ transcript predicts a poor overall survival (OS) rate in the unclassified patients or patients with LGG or GBM in a Kaplan-Meier analysis under a maximal risk condition (Figure 2B). To further confirm the prognostic significance of H2AFJ in GBM, we utilized three Gene Expression Omnibus (GEO) datasets GSE13041 [24], GSE4412 [25], and GSE42669 [26] to perform another Kaplan-Meier analysis of H2AFJ gene expression. The data showed that the elevation of the H2AFJ transcript was significantly $(p<0.01)$ associated with a poor survival rate in glioma patients in those three datasets (Figure 2B). Furthermore, the transcriptional profiling revealed that the expression of H2AFJ in GBM, grade IV glioma and recurrent tumors is more abundant than that in LGG, grade II/III glioma and primary tumors, respectively, in TCGA LGG/GBM database (Figure 2C). Whereas the upregulation of H2AFJ was evident for brain tumors from patients with age equal to or over 64 years, its mRNA levels appeared to be no different in brain tumors from female and male patients (Figure S3A,B). Again, these findings indicated that H2AFJ upregulation may refer to a poor prognosis and associate with a poor therapeutic response in brain tumors.

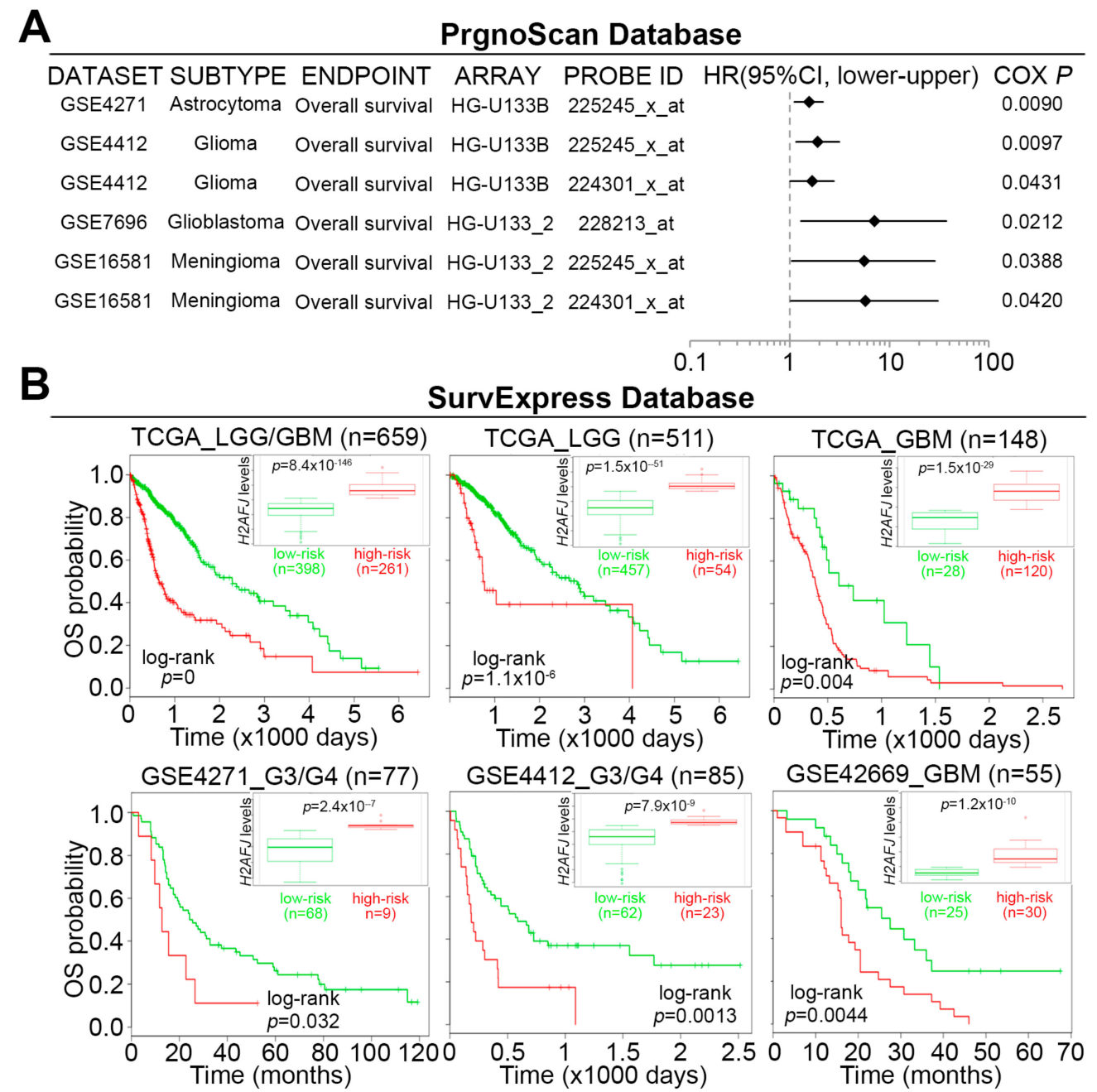

Figure 2. Cont. 


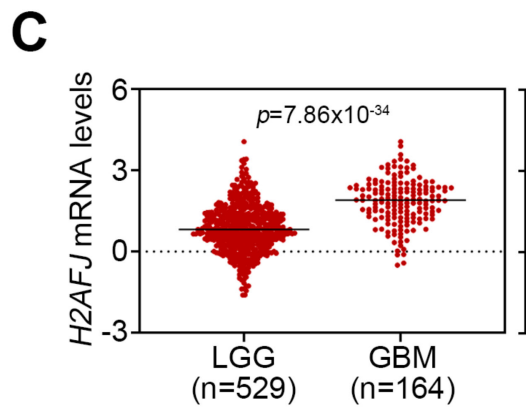

\section{TCGA_LGG/GBM}

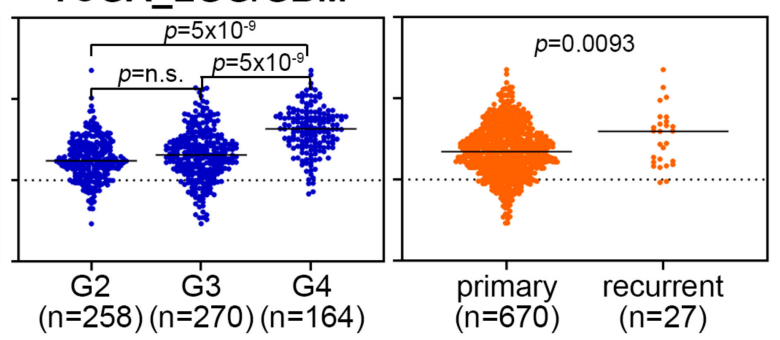

Figure 2. H2AFJ acts as a poor prognostic marker in brain tumors, particularly GBM. (A) A meta-analysis of H2AFJ in low-grade glioma and GBM using the PrognoScan database (left). A forest plot (right) was used to present the hazard ratio (HR) distribution in the $95 \%$ confidence interval (CI) estimated by Cox regression against H2AFJ transcript levels (high vs. low) in the enrolled cohorts. (B) Prognostic estimation of H2AFJ expression using the SurvExpress program under the condition of overall survival (OS) probability in low-grade glioma (LGG)/GBM, LGG or GBM cohorts from TCGA and LGG or GBM cohorts from three GEO datasets (GSE4271, GSE4412, and GSE42669). The inserts indicate the mRNA levels of H2AFJ in the low- and high-risk groups. (C) Dot plots for the transcriptional profiling of $\mathrm{H} 2 \mathrm{AFJ}$ in clinical tissues derived from LGG and GBM, different histological grades, or primary and recurrent tumors using TCGA LGG/GBM database. The bars indicate the mean of H2AFJ mRNA levels in each group. The statistical significance was analyzed by Student t-test (left and right) or one-way ANOVA and Turkey's post-hoc test (middle). The symbol "n.s." denotes not significant.

\subsection{H2AFJ Expression Determines TMZ, not Radiation, Effectiveness in GBM.}

To ascertain the association of H2AFJ expression with the therapeutic effectiveness of radiation and TMZ on GBM, we next validated this phenomenon in GBM cell lines. We found that the endogenous mRNA levels of H2AFJ and MGMT were causally associated (Figure 3A) and significantly ( $p=0.05$ ) correlated with the $50 \%$ inhibitory concentration $\left(\mathrm{IC}_{50}\right)$ of $\mathrm{TMZ}$ (Figure $3 \mathrm{~B}$ ) but not the cytotoxic effectiveness of radiation (Figure 3C,D) in the analyzed GBM cell lines. Whereas H2AFJ knockdown sensitized D54MG glioma cells to TMZ treatment (Figure 3E), H2AFJ overexpression rendered T98G glioma cells more resistant to TMZ treatment (Figure 3F).
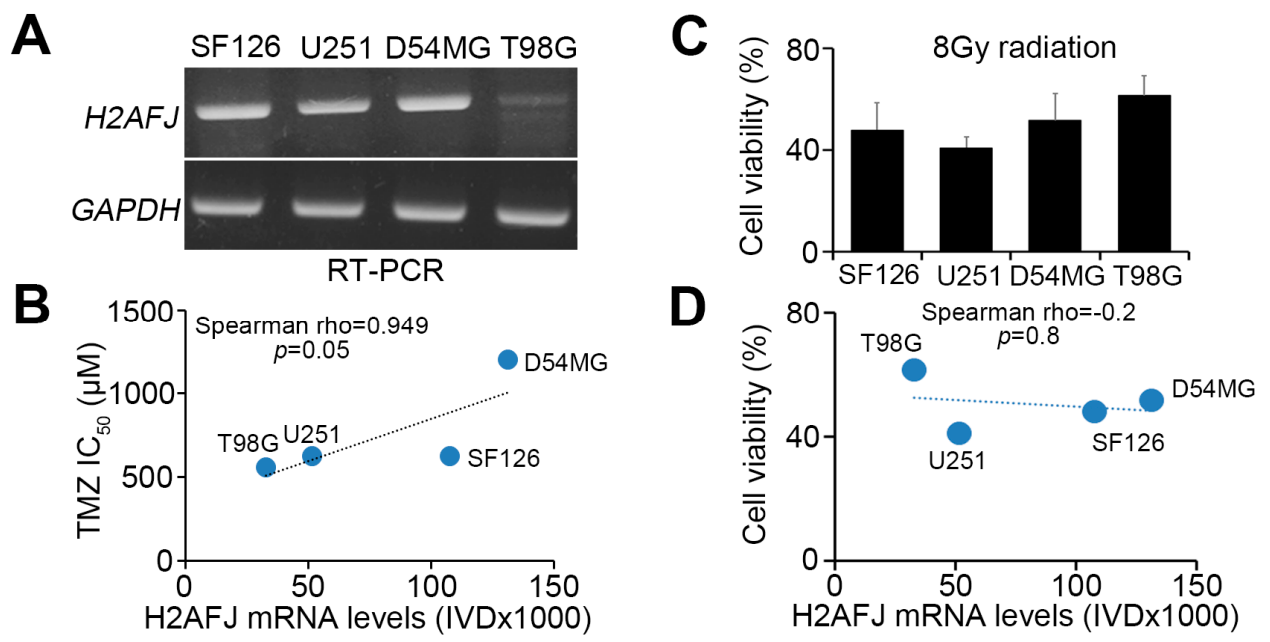

Figure 3. Cont. 

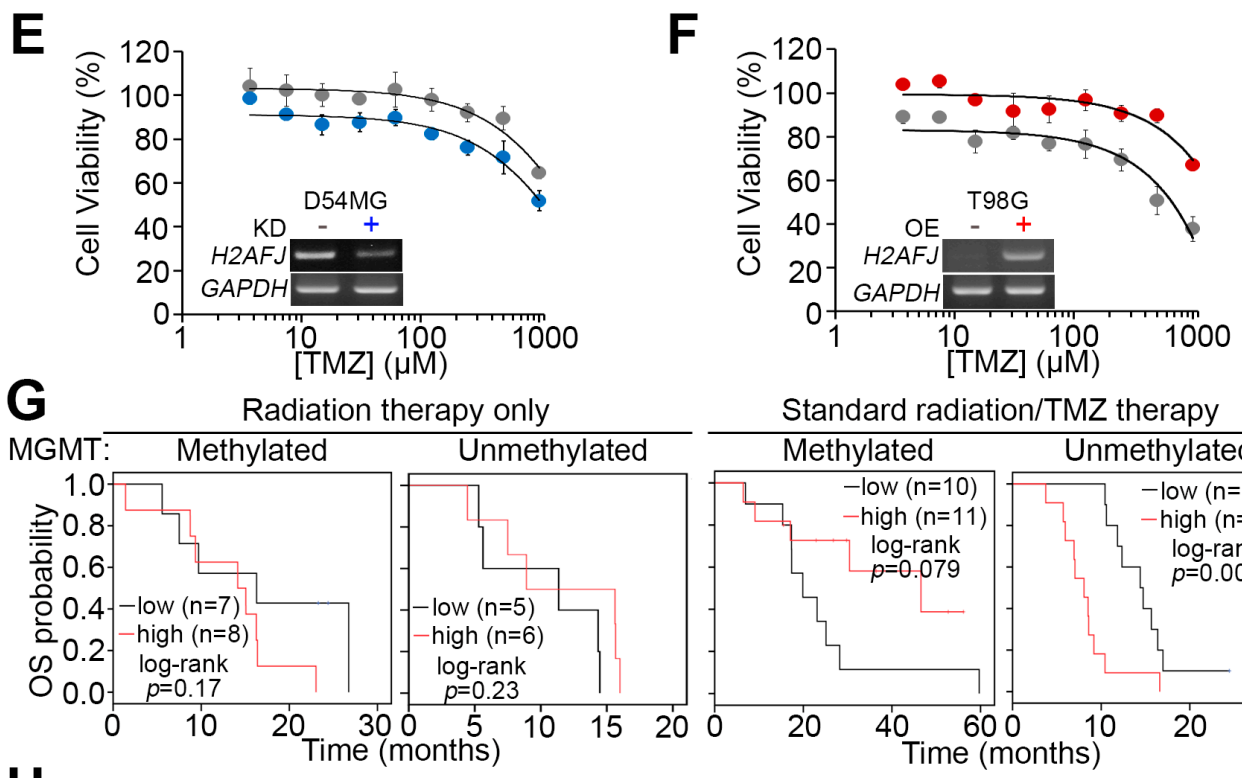

Standard radiation/TMZ therapy

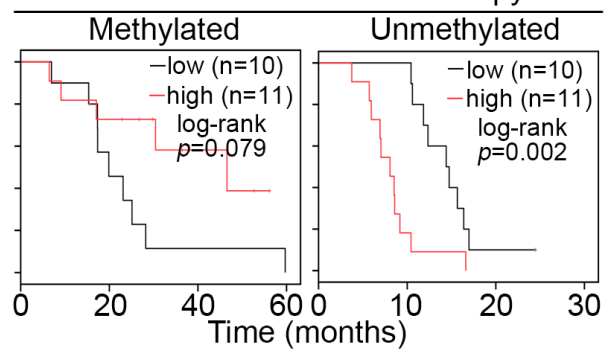

\section{H}

TCGA GBM patients (wild-type IDH1/non-G-CIMP)
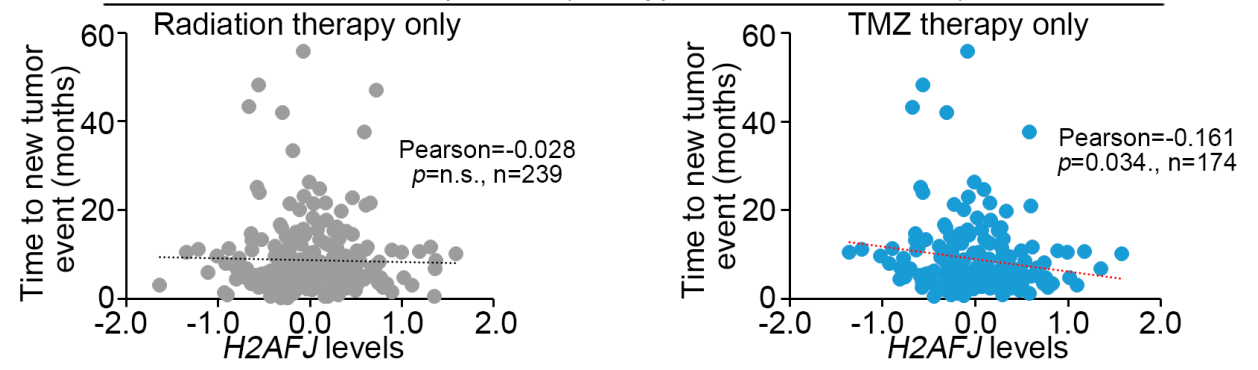

Figure 3. H2AFJ upregulation desensitizes GBM cells to TMZ treatment. (A) RT-PCR analysis of $H 2 A F J$ and GAPDH expression in different GBM cell lines. (B) Scatter plots for the correlation between $\mathrm{H} 2 \mathrm{AFJ}$ expression and $\mathrm{TMZ} \mathrm{IC}_{50}$ concentrations in various GBM cells. (C) Cell viability of the detected GBM cells after 24-h exposure to radiation at $8 \mathrm{~Gy}$. The count of remaining viable cells after radiation treatment was normalized with the cell number of the untreated group in each detected GBM cell line. The data from three independent experiments are presented as the mean \pm SEM. (D) Scatter plots for the correlation between H2AFJ mRNA levels and cell viability of the detected GBM cells posttreatment with radiation at $8 \mathrm{~Gy}$ for $24 \mathrm{~h}$. A non-parametric Spearman correlation test was used to estimate statistical significance in B and D. (E,F) Scatchart plots for the cell viability of D54MG cells (E) with or without H2AFJ knockdown (insert) and T98G cells (F) with or without H2AFJ overexpression (insert) after TMZ treatment for four days at the indicated TMZ concentrations. In A, E and F, GAPDH was used as an internal control for RT-PCR experiments. (G) Kaplan-Meier analysis of overall survival probability associated with H2AFJ gene expression in MGMT-unmethylated and -methylated GBM patients undergoing radiation therapy or standard TMZ/radiation therapy. (H) Scatter plots for H2AFJ transcripts and time to a new tumor event after radiation or TMZ therapy in GBM patients who were diagnosed to be wild-type IDH and non-G-CIMP. The statistical significance was estimated by Pearson's correlation test. The symbol "n.s." denotes not significant.

Since the methylation of the MGMT promoter has been correlated with a favorable TMZ response, we next examined the prognostic significance of $\mathrm{H} 2 \mathrm{AFJ}$ in patients receiving radiation therapy combined with or without TMZ treatment and in GBM with or without MGMT methylation using the GSE7696 dataset. In patients receiving radiation therapy (RT) only, regardless of MGMT methylation status, H2AFJ gene expression was not significantly correlated with an overall survival probability of he enrolled GBM patients in a Kaplan-Meier analysis (Figure 3G). In patients receiving combined treatment of radiation and TMZ followed by TMZ therapy only, H2AFJ upregulation significantly $(p<0.01)$ predicted a poor overall survival rate in patients with MGMT-unmethylated GBM (Figure 3G). 
In contrast, in patients with MGMT-methylated GBM, H2AFJ expression was causally associated with overall survival time after treatment with radiation and TMZ therapy (Figure 3G). Similarly, in TCGA cohorts with IDH1 wild-type GBM without glioma CpG island methylation phenotype (G-CIMP), the increased mRNA levels of H2AFJ was significantly $(p=0.034)$ correlated with a shorter time to new tumor event after patients receiving TMZ, not radiation, therapy (Figure $3 \mathrm{H}$ ). Therefore, H2AFJ may serve a predictive biomarker for the TMZ effectiveness in GBM.

\subsection{H2AFJ Upregulation Associates with the Progression of Proneural-Mesenchymal Transition and the} Activation of TNF $\alpha-N F-\kappa B$ and IL6-STAT3 Signaling Pathways in GBM

To realize the possible mechanism for H2AFJ-promoted TMZ resistance in GBM, we next performed an in silico analysis. We performed a Pearson correlation test for the co-expression of H2AFJ gene with other somatic genes in TCGA GBM tissues (Figure 4A) from cBioPortal. We next analyzed this gene signature by Gene Set Enrichment Analysis (GSEA). The GSEA results showed that this H2AFJ-related gene signature was significantly $(p<0.001)$ correlated with the upregulation of epithelial-mesenchymal transition (EMT), TNF-alpha/NF-kB and IL-6/STAT3 pathways (Figure 4B). Because the induction of proneural-mesenchymal transition (PMT) has been shown to be similar to EMT and correlated with a mechanism for TMZ resistance in GBM cells [20], we next analyzed the correlation between the expression of the H2AFJ gene and PMT signature genes, which include WT1, TGFBR2, LYN, CD44, YKL40, and BCL2A1, in the GBM tissues from TCGA. The data revealed that H2AFJ gene expression was highly correlated with the mRNA levels of PMT signature genes (Figure 4C). Whereas H2AFJ knockdown in D54MG cells repressed the gene expression of CD44 (Figure 4D), H2AFJ overexpression in T98G cells enhanced the transcription of CD44 (Figure 4E). In addition, the mRNA levels of TNF-alpha and H2AFJ (Figure 4F), as well as interleukin-6 and H2AFJ (Figure 4G), were shown to be positively correlated in the TCGA GBM database. Artificially silencing the expression of H2AFJ predominantly repressed the mRNA levels of TNF-alpha and interleukin-6 in D54MG glioblastoma cells (Figure 4H), whereas the forced gene expression of ectopic H2AFJ dramatically enhanced the expression of TNF-alpha and interleukin-6 in T98G glioblastoma cells (Figure 4I). Moreover, a luciferase-based reporter assay demonstrated that H2AFJ knockdown reduced but overexpression elevated the transcription factor activities of NF- $\mathrm{KB}$ (Figure $4 \mathrm{~J}$ ) and STAT3 (Figure 4K) in the tested D54MG and T98G cells, respectively.

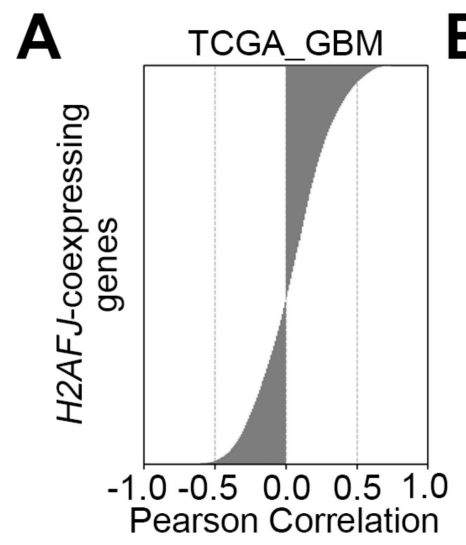

B

Gene set enrichment analysis

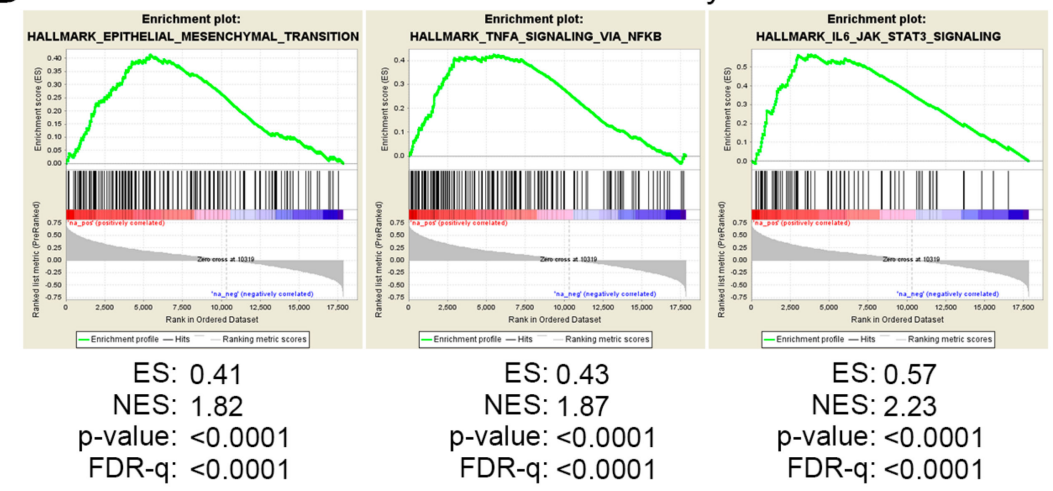

Figure 4. Cont. 

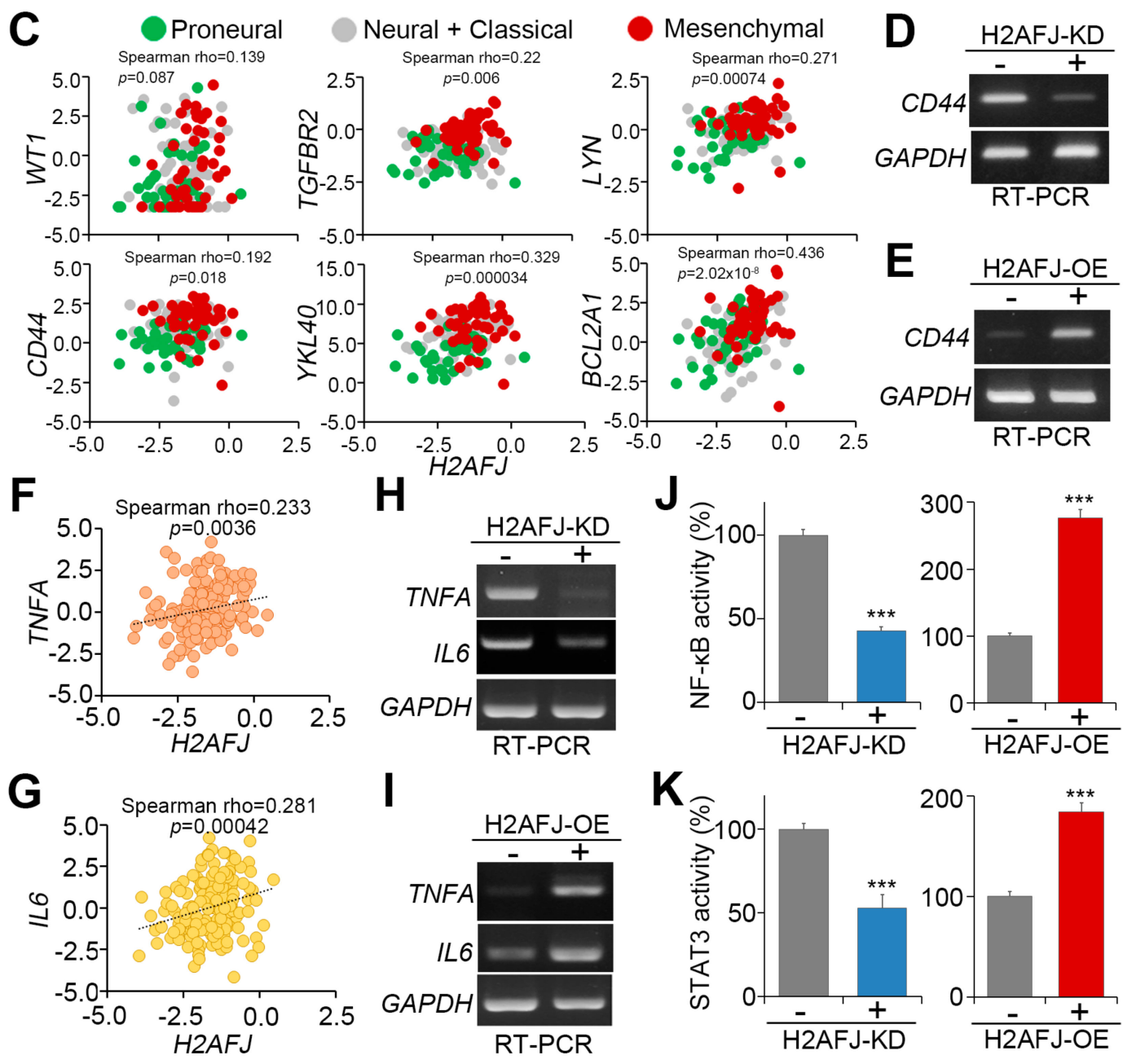

Figure 4. H2AFJ upregulation is associated with proneural-mesenchymal transition and activation of TNF $\alpha / N F-\kappa B$ and IL-6/STAT3-related pathways. (A) Histogram for the results from Pearson's correlation test of the transcriptional profile for H2AFJ and other somatic genes in TCGA GBM tissues. (B) Snapshot of gene set enrichment analysis (GSEA) results showing as enrichment plots for epithelial-mesenchymal transition, TNF $\alpha / \mathrm{NF}-\mathrm{kB}$ and IL-6/STAT3 gene sets using the H2AFJ-related gene signature in A. (C) Scatter plots for the correlation of transcriptional profiles among $H 2 A F J$ and proneural-mesenchymal transition-associated markers (WT1, TGFBR2, LYN, CD44, YKL40, and $B C L 2 A 1)$ in GBM tissues from TCGA. (D,E) RT-PCR analysis for the mRNA levels of CD44 and GAPDH in D54MG cells without or with H2AFJ knockdown (KD, D) and T98G cells without or with H2AFJ overexpression $(\mathrm{OE}, \mathbf{E})$. (F,G) Scatter plots for the correlation among the gene expression of TNF-alpha (TNFA, F), interleukin-6 (IL6, G) and H2AFJ in the TCGA GBM database. In C, F, G, the statistical significance was analyzed by Spearman's correlation test. (H,I) RT-PCR analysis for the mRNA levels of TNFA, IL6, and GAPDH in D54MG cells without or with H2AFJ knockdown (KD, H) and T98G cells without or with $H 2 A F J$ overexpression (OE, I). In D, E, H and I, GAPDH was used as an internal control for RT-PCR. (J,K) Histograms represent the results of luciferase-based reporter assays for NF- $\mathrm{KB}(\mathrm{J})$ and STAT3 (K) activities in D54MG cells without (non-silencing control, NS) or with H2AFJ knockdown (KD) and T98G cells without (vector control, VC) or with H2AFJ overexpression (OE). The data from three independent experiments are presented as the mean \pm SEM. "**** denotes statistical significance at $p<0.001$ for a non-parametric Mann-Whitney test. 
By using the TCGA database, we found a significantly $(p<0.0001)$ positive correlation among the mRNA levels of H2AFJ, PMT geneset, TNF $\alpha$-NF-KB geneset, and IL6-STAT3 geneset in GBM tissues (Figure 5A). Moreover, Kaplan-Meier analyses under a maximal risk condition demonstrated that high-level expression of PMT, TNF $\alpha-N F-k B$, and IL6-STAT3 genesets refers to a poor overall survival probability TCGA GBM patients (Figure 5B). Robustly, compared to the combination of low-level H2AFJ with low-level expression of PMT, TNF $\alpha$-NF-kB, or IL6-STAT3 geneset, as well as other combinations, the scenario of combining high-level of $H 2 A F J$ with high-level expression of PMT, TNF $\alpha$-NF- $\mathrm{KB}$, or IL6-STAT3 geneset predicted a worse prognosis (Figure 5C) and shorter overall survival time (Figure 5D) in TCGA GBM patients. In TMZ-receiving patients with GBM harboring wild-type IDH1 and non-G-CIMP, the combined expression of H2AFJ with PMT, TNF $\alpha-N F-\kappa B$, or IL6-STAT3 geneset exhibited an inverse correlation with time to new tumor event after the treatment (Figure 5E). It might indicate that the upregulation of H2AFJ accompanied by the activation of PMT, TNF $\alpha$-NF-KB, and IL6-STAT3 pathways is associated with the mechanism for TMZ resistance in GBM.

\subsection{Pharmaceutical Inhibition of NF- $\kappa B$ and HDAC3 Provides a New Strategy for Overcoming TMZ-Insensitive GBM with H2AFJ Upregulation}

To combat the TMZ resistance due to H2AFJ upregulation in GBM, we next performed an in silico genomic-based drug discovery using the Cancer Therapeutics Response Portal (CTRP) database [27,28]. The Pearson's correlation test against H2AFJ mRNA levels and the $\mathrm{IC}_{50}$ AUC values of deposited compounds showed that the $\mathrm{IC}_{50}$ concentrations of tacedinaline, a selective inhibitor for class I histone deacetylase (HDAC) 1, 2, 3, and 8, and ML029, an inhibitor for NF-KB negatively correlates with H2AFJ mRNA levels in a panel of glioma cell lines (Figure 6A,B and Figure 44 ). In contrast, the $\mathrm{IC}_{50}$ concentrations of TMZ appeared to be positively correlated with $\mathrm{H} 2 \mathrm{AFJ}$ transcriptional profile in the detected glioma cells (Figure 6A,B and Figure S4). The negative correlation of H2AFJ expression with tacedinaline or ML029 IC 50 concentrations but its positive correlation with $\mathrm{TMZ} \mathrm{IC} \mathrm{C}_{50}$ concentrations was evident for KNS60 and U251 glioma cells (Figure 6B and Figure S4). Furthermore, we performed transcriptional profiling of HDAC family in order to identify a highly co-expressing HDAC with $H 2 A F J$ in GBM (Figure 6C). We found that the expression of $H 2 A F J$ and $H D A C 3$ is significantly $(p<0.001)$ and positively correlated in the primary tumors of TCGA GBM database (Figure 6D). While HDAC3 upregulation was correlated with a poor prognosis, the scenario of combining high-level expression of H2AFJ and HDAC3 predicted a worse overall survival probability in the TCGA GBM patients in Kaplan-Meier analyses using a maximal risk condition (Figure 6E). Accordingly, in comparison with other combinations, the combination of high-level H2AFJ and HDAC3 expressions was significantly $(p<0.01)$ associated with a shorter overall survival time of GBM patients (Figure 6F). Moreover, the combined expression of $H 2 A F J$ and HDAC3 was appeared to be inversely correlated with the time to new tumor event after TMZ therapy in the wild-type IDH1/non-G-CIMP GBM patients (Figure 6G). Finally, the computational simulation using Pathway Commons Network Visualizer revealed a putatively inter-molecular interaction among H2AFJ with HDAC3, TNF $\alpha / \mathrm{NF}-\mathrm{kB}$, and IL6/STAT3 (Figure 6H). These findings may provide tacedinaline or ML029 as an alternative strategy to treat TMZ-insensitive GBM with H2AFJ upregulation. 

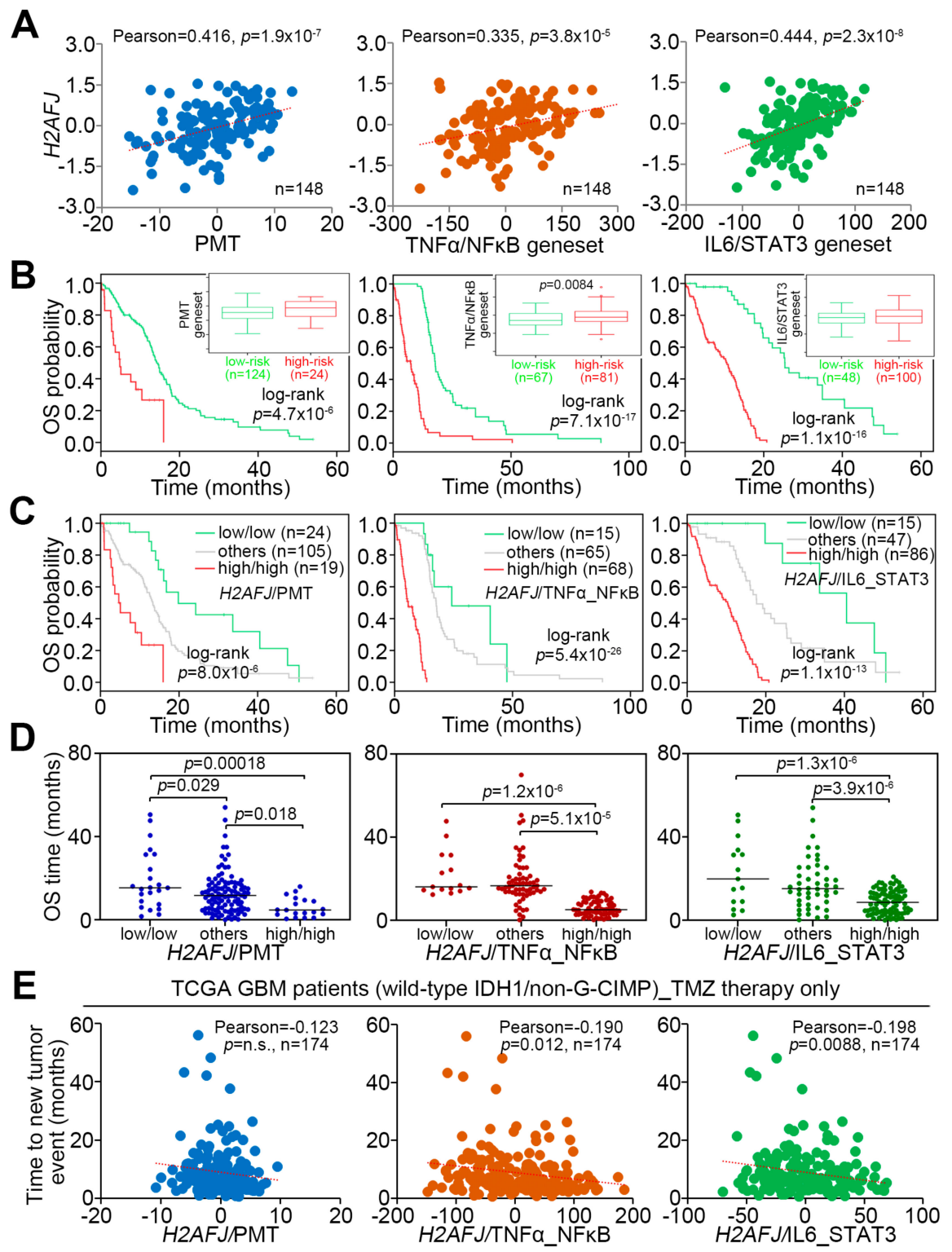

Figure 5. The signature of combing high-level H2AFJ transcripts with high-level PMT geneset, $\mathrm{TNF} \alpha / \mathrm{NF}-\kappa \mathrm{B}$ geneset or IL-6/STAT3 geneset expression correlates with poor responsiveness to TMZ therapy in patients with GBM harboring wild-type IDH1 and non-G-CIMP. (A) Scatter plots for the correlation of mRNA levels determined by RNA sequencing technique among H2AFJ, PMT geneset, TNF $\alpha / \mathrm{NF}-\kappa \mathrm{B}$ geneset or IL-6/STAT3 geneset in primary tumors derived from the TCGA GBM database. (B,C) Kaplan-Meier analyses using overall survival (OS) probability for the low and high-risk populations defined by SurvExpress program under a maximal risk condition in accordance with the transcriptional levels of PMT geneset, TNF $\alpha / N F-\kappa B$ geneset or IL-6/STAT3 geneset combined without (B) or with (C) H2AFJ mRNA levels in TCGA GBM database. Insets represent the mRNA levels of the factors in low and high-risk populations. In $\mathrm{C}$, others denote the low/high and high/low expression levels. (D) Dot plots represent overall survival time in the three populations stratified in $\mathrm{C}$ according to the transcriptional profiles of H2AFJ, PMT geneset, TNF $\alpha / N F-\kappa B$ geneset or IL-6/STAT3 geneset in primary tumors derived from the TCGA GBM database. The bars indicate the mean of overall survival time in each group. One-way ANOVA and Turkey's post-hoc test was used to estimate the statistical significance. (E) Scatter plots for the correlation of time to new tumor event with the H2AFJ mRNA levels combined with the transcriptional profiles of PMT geneset, TNF $\alpha / N F-\kappa B$ geneset or IL-6/STAT3 geneset in the TCGA IDH1 wild-type/non-G-CIPM GBM patients receiving TMZ therapy. The statistical significance was analyzed by Pearson's correlation test. The symbol "n.s." denotes not significant. 

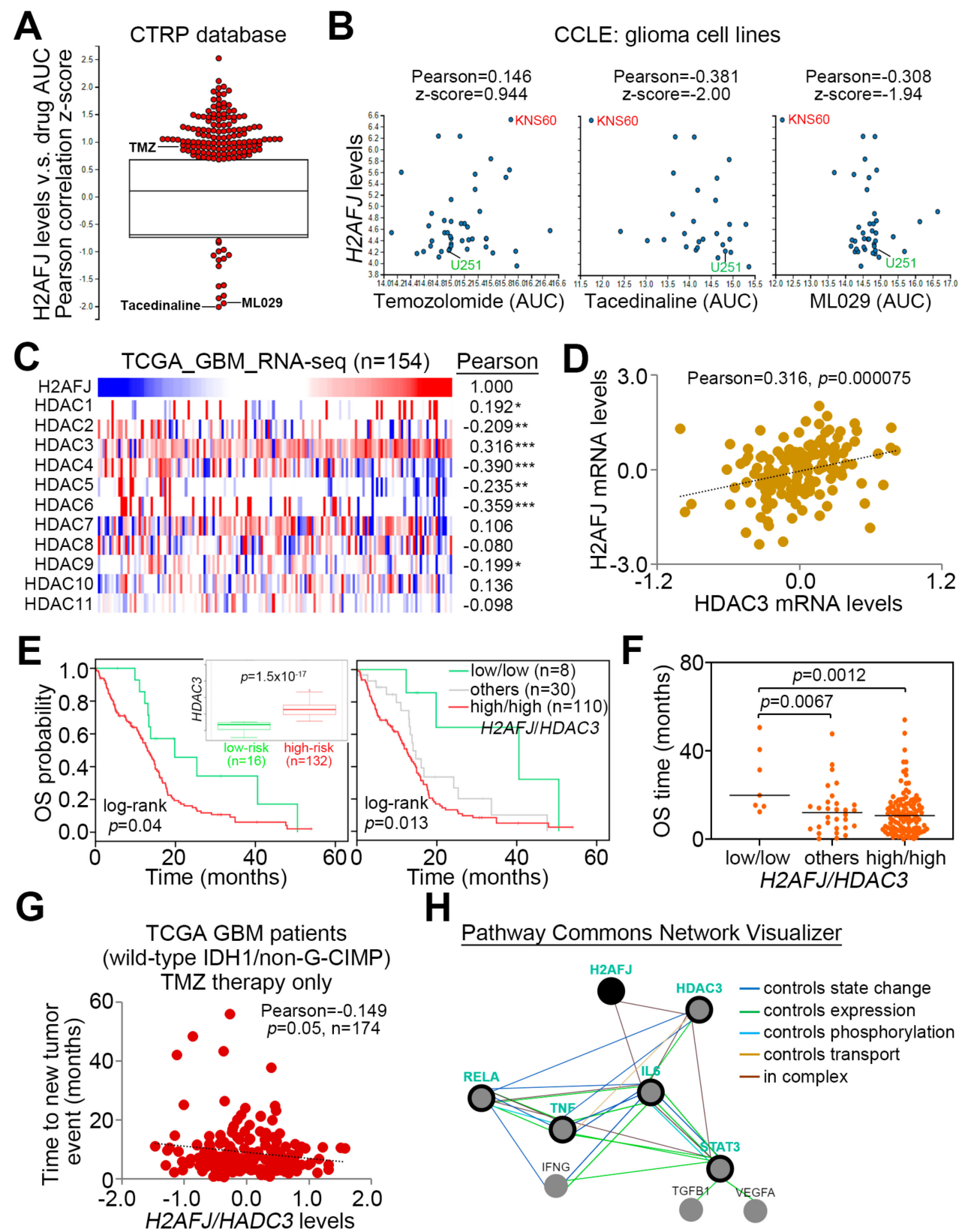

H

Pathway Commons Network Visualizer

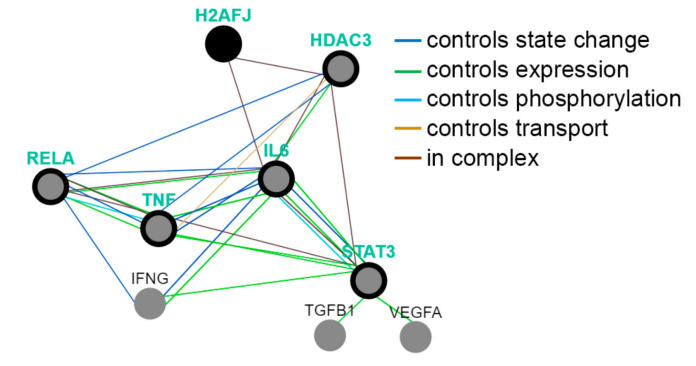

Figure 6. (A) Box/dot plot for the z-score derived from Pearson's correlation test for the transcriptional profile of $\mathrm{H} 2 \mathrm{AFJ}$ and the AUC values of drug $\mathrm{IC}_{50}$ concentrations in glioma cell lines using CTRP database. AUC is abbreviated from the area under the curve of the receiver operating characteristic curve. (B) Scatter plots represent the results of Pearson's correlation test for the association of H2AFJ mRNA levels with the AUC values of temozolomide, tacedinaline or ML029 $\mathrm{IC}_{50}$ concentrations in glioma cell lines using the Cancer Cell Line Encyclopedia (CCLE) database. (C) Heatmap for the transcriptional profiles of $\mathrm{H} 2 \mathrm{AFJ}$ and HDAC subfamily determined by RNA sequencing method in the TCGA GBM database. The results of Pearson's correlation test against the transcriptional profiles of H2AFJ and the all listed genes are shown next to the heatmap. The symbols ${ }^{*}{ }^{* *}$ and ${ }^{* * *}$ denote the statistical significance at $p<0.05, p<0.01$, and $p<0.001$ in Pearson's correlation test. (D) Scatter plot represents the correlation for the transcriptional profile of H2AFJ and HDAC3 in the TCGA GBM database. (E) Kaplan-Meier analyses using overall survival (OS) probability for the low and high-risk populations defined by SurvExpress program under a maximal risk condition in accordance with the transcriptional profile of HDAC3 combined without (left) or with (right) H2AFJ mRNA levels in TCGA 
GBM database. Insets represent the mRNA levels of HDCA3 in low and high-risk populations. In the right figure, others denote the low/high and high/low expression levels of H2AFJ and HDAC3. (F) Dot plots represent overall survival time in the three population stratified in E according to the transcriptional profiles of H2AFJ and HDCA3 in primary tumors derived from the TCGA GBM database. The bars indicate the mean of overall survival time in each group. One-way ANOVA and Turkey's post-hoc test was used to estimate the statistical significance. (G) Scatter plots for the correlation of time to a new tumor event with the transcriptional profile of H2AFJ/HDAC3 in TCGA IDH1 wild-type/non-G-CIPM GBM patients receiving TMZ therapy. The statistical significance was analyzed by Pearson's correlation test. $(\mathbf{H})$ The network of inter-molecular interactions among H2AFJ, HDAC3, TNF $\alpha /$ NF-kB (RELA), and IL-6/STAT3 using Pathway Commons Network Visualizer program. The relationships of inter-molecular interaction are shown in the inserted panel.

\section{Discussion}

Based on gene expression patterns and clinical characteristics, GBM has been classified into four subtypes: Proneural, neural, classical, and mesenchymal [6]. Among these subtypes, mesenchymal-type GBM exhibits the most aggressive phenotypes (e.g., TMZ resistance), and therefore, results in a worse prognosis in GBM patients [6,29]. Here, we show that H2AFJ differs from other H2A subfamily members in that it is highly expressed by mesenchymal-type GBM tissues and serves as a predictive factor for TMZ effectiveness in GBM patients. Moreover, artificially silencing H2AFJ sensitized but overexpressing H2AFJ desensitized GBM cells to the cancericidal effect of TMZ. In GBM patients who lack methylation at the MGMT promoter region, a GBM subpopulation presumably insensitive to TMZ treatment [30,31], we found that H2AFJ upregulation is able to further differentiate a poorer TMZ-responsive subgroup from those patients. In addition, our data showed that $H 2 A F J$ upregulation is extensively detected in GBM tissues with wild-type IDH1, another indicator for a poor TMZ response [10,12]. Notably, a meta-analysis revealed that $H 2 A F J$ upregulation is a poor prognostic marker in brain tumors, irrespective of pathological grade. Based on these findings, we propose that the combination of H2AFJ levels with MGMT and/or IDH1 features is likely able to more precisely identify brain tumor patients who will have good outcomes after TMZ therapy.

Proneural-mesenchymal transition (PMT) has been identified as a process of cancer progression and is related to the mechanism for TMZ resistance in GBM [20]. The induction of PMT post-radiation therapy through the activation of the NF- $\mathrm{KB}$ and STAT3 pathways by inflammatory agents, such as IL-6, TNF- $\alpha$, etc., has been found in GBM [32-34]. Moreover, IL-6 overexpression has been identified as a marker of malignancy in GBM [35]. Here, we showed that H2AFJ expression was causally associated with the PMT process, as judged by a positive correlation between $H 2 A F J$ and PMT signature gene mRNA levels in GBM tissues. Moreover, we found that $H 2 A F J$ knockdown dramatically suppressed IL-6 expression and NF- $\mathrm{kB}$ activity in GBM cells. Although a more comprehensive mechanism by which H2AFJ regulates the IL-6-NF- $\mathrm{KB}$ axis to modulate PMT progression should be further explored in GBM, our findings may be the first to document that the upregulation of $H 2 A F J$, but not of other H2A subfamily members, is associated with the molecular mechanism for PMT progression, which confers TMZ resistance in GBM.

NF- $\mathrm{KB}$ has been identified as a transcriptional regulator of MGMT [31,36]. The overexpression of ectopic p65 or high constitutive NF- $\mathrm{kB}$ activity has been shown to elevate MGMT and thereby enhance chemoresistance to alkylating agents in detected cells [37]. In contrast, the inhibition of NF- $\mathrm{kB}$ by the small molecule inhibitor BAY 11-7082 or by siRNA-mediated gene silencing reduced MGMT levels and thereby sensitized glioma stem-like cells to TMZ treatment [30,32]. Here, we found that $\mathrm{H} 2 \mathrm{AFJ}$ expression causally modulates the activity of NF-KB in GBM cells. Based on these findings, we hypothesize that H2AFJ acts as an upstream regulator of NF- $\mathrm{kB}$-mediated MGMT expression and PMT induction, which play crucial roles in conferring TMZ resistance in GBM. Moreover, an in silico simulation using Pathway Commons Network Visualizer program demonstrated that the interaction of HDAC3 with H2AFJ may epigenetically regulate the elevation of the IL-6 transcription, thereby enhancing the activity of IL-6/STAT3 signaling axis which subsequently triggers the activation of 
TNF- $\alpha / N F-\kappa B$ pathway via elevating the gene expression of TNF- $\alpha$. Although our results revealed that the gene expression of IL- 6 and TNF- $\alpha$ is causally affected after H2AFJ knockdown and overexpression in the detected cells, further experiments are still needed to clarify the role of HADC3 in regulating $\mathrm{H} 2 \mathrm{AFJ}$ activity by a post-translational modification of lysine acetylation and identify if the genomic DNA of IL-6, as well as other related molecules, is recruited as the component of H2AFJ-associated nucleosome in GBM.

Post-translational modifications (PTMs) of histones by the epigenetic enzymes, e.g., HDACs, is essential for their protein functions in regulating gene expression during normal cell development $[38,39]$ and cancer progression $[40,41]$. Since histone variants are highly conserved between different species, their functions cannot be accomplished by canonical histones [42]. The altered expression of H2As has been found in cancers and resulted in different consequences. Whereas the H2AFX and H2AFY are considered as tumor suppressors, H2AFZ exhibits an oncogenic function [43]. Here, we further show that $\mathrm{H} 2 \mathrm{AFJ}$ likely serves as a driver in promoting TMZ resistance and may be associated with the mesenchymal differentiation in GBM. Therefore, targeting H2AFJ functions could be a new strategy to overcome the most aggressive mesenchymal-GBM. Nevertheless, the use of additional genetic brain tumor profiling resources (e.g., longitudinal tumor trajectories [44]) and further experimental validation in diverse genotypic context (IDH1 mutant cell models, etc.) is still needed to clarify whether or how the phenotype of H2AFJ upregulation is linked to a specific clonal or subclonal evolution of a clinically-relevant genotype of brain tumor progression and therapeutic resistance. In addition, further in vitro and in vivo experiments (i.e., utilizing a spectrum of patient-derived models and tumor stem cell contexts) are also required for validating the potential therapeutic significance of elevated H2AFJ in glioblastoma resistance working toward patient stratification.

\section{Materials and Methods}

\subsection{Clinical and Molecular Data for Patients}

Raw data for the pathologic information and prognostic value of $H 2 A F J$ were downloaded from the PrognoScan database (http://www.abren.net/PrognoScan/). Datasets with a statistical $p$-value $<0.05$ in a Cox regression analysis were included in a meta-analysis for H2AFJ gene expression. Transcriptional profiling of H2AFs was obtained from The Cancer Genome Atlas (TCGA, data released in Jan 2016) and Gene Expression Omnibus (GEO) databases and subjected to statistical analysis for the differential display of their mRNA in subgroups. A Kaplan-Meier analysis and the differential display for H2AFJ gene expression were performed on the SurvExpress website or using SPSS software.

\subsection{Cell Lines and Cell Culture Conditions}

GBM cell lines obtained from the American Type Culture Collection (ATCC, Manassas, VA, USA) were maintained in conditioned media. We maintained 293T cells in DMEM supplemented with $10 \%$ fetal bovine serum (FBS). Cells were incubated at $37{ }^{\circ} \mathrm{C}$ with $5 \% \mathrm{CO}_{2}$. All cells were routinely authenticated on the basis of short tandem repeat (STR) analysis, morphologic and growth characteristics and mycoplasma detection.

\subsection{MTT Assay}

Cells $\left(5 \times 10^{4} / \mathrm{mL}\right)$ were seeded into a 96-well culture plate. After incubation, $10 \mu \mathrm{L}$ of MTT (3-(4,5-dimethylthiazol-2-yl)-2,5-diphenyltetrazolium bromide, molecular probe, (Invitrogen, Carlsbad, CA, USA) stock solution was added to each well. The conversion of MTT to formazan by viable cells was performed at $37^{\circ} \mathrm{C}$ for $4 \mathrm{~h}$. After the reaction, $100 \mu \mathrm{L}$ of DMSO solution was added to each well to solubilize the formazan precipitates. The levels of formazan were determined by optical density at 540 $\mathrm{nm}$ using an ELISA reader for calculating cell survival rates. 


\subsection{Plasmid Construction}

The gene encoding H2AFJ was amplified from human cDNA (Invitrogen) using a standard polymerase chain reaction (PCR) procedure with paired primers and subcloned into the pDONR221 vector. The identities of individual clones were verified via double-strand plasmid sequencing. The pDONR221 plasmid was recombined with the destination vector pLenti6.3/V5-DEST in 293T cells to create packaged lentiviral particles. A commercially available plasmid, pLenti/GFP, sharing the same backbone (Invitrogen) was used as a control. The recombinant lentiviruses in the culture medium were harvested, concentrated using a Lenti-X Concentrator (Clontech, Mountain View, CA, USA), and then titrated by determining the viral RNA genome content with a Lenti-X qRT-PCR titration kit (Clontech) according to the manufacturer's instructions.

\subsection{Lentivirus-Driven shRNA Infection}

Lentiviral shRNA constructs were purchased from Open Biosystems. Lentiviruses were produced by cotransfecting the shRNA-expressing vector with the pMDG and $\mathrm{p} \triangle 8.91$ constructs into 293T cells using a calcium phosphate transfection kit (Invitrogen). After incubation for 48-72 h, the media were collected as viral stocks. Cells (50\% confluence) grown on six-well plates were incubated in fresh media containing $5 \mu \mathrm{g} / \mathrm{mL}$ polybrene (Santa Cruz Biotechnology, Santa Cruz, CA, USA) before infection overnight with a lentiviral viral particle-driven control or candidate gene shRNA at 2-10 multiplicity of infection (MOI). To select cells stably expressing the control or candidate gene shRNA, cells were further cultivated in the presence of puromycin $(10 \mu \mathrm{g} / \mathrm{mL})$ for $24 \mathrm{~h}$. Cell lysates from the puromycin-resistant cells were subsequently subjected to PT-RCR analysis to confirm the efficiency of gene knockdown.

\subsection{Reverse Transcription PCR (RT-PCR)}

Total RNA was extracted from cells using a TRIzol extraction kit (Invitrogen). Aliquots (5 $\mu \mathrm{g})$ of total RNA were treated with M-MLV reverse transcriptase (Invitrogen) and then amplified with Taq-polymerase (Protech) using paired primers (for H2AFJ, forward-CTGGATGTTGGGCAGGACG and reverse-GAAACAGGGCGGCAAAGTGC, for MGMT, forward-TCATCCCGTGCCACAGA and reverse-AGCGGTGCCTCCACG, for TNF, forward-TGGCCCAGGCAGTCAGAT and reverseGCAGCCTTGGCCCTTGAA, for IL6, forward-CTGGATGTTGGGCAGGACG and reverseGAAACAGGGCGGCAAAGTGC, and for GAPDH, forward-AGGTCGGAGTCAACGGATTTG and reverse-GTGATGGCATGGACTGTGGTC).

\subsection{Irradiation Treatment and Cell Viability Analysis}

Irradiation was performed with $6 \mathrm{MV}$ X-rays using a linear accelerator (Digital M Mevatron Accelerator, Siemens Medical Systems, Ann Arbor, MI, USA) at a dose rate of 8 Gy/min. An additional $2 \mathrm{~cm}$ of a tissue-equivalent bolus was placed on the top of the plastic tissue-culture flasks to ensure electronic equilibrium, and $10 \mathrm{~cm}$ of tissue-equivalent material was placed under the flasks to obtain full backscatter. After IR treatment, cells were centrifuged and resuspended with an appropriate amount of PBS. For the cell viability assay, $20 \mu \mathrm{L}$ of the cell suspension was mixed with $20 \mu \mathrm{L}$ of Trypan blue solution $(0.4 \%$ in PBS). The stained cells were placed on a hemocytometer, and the blue-stained cells were counted as nonviable under a microscope.

\subsection{Luciferase Reporter Assay}

Luciferase reporter vectors containing NF- $\mathrm{BB}$ or sis-inducible element (SIE) response elements within the promoter region were purchased from Promega and utilized to estimate the activity of NF- $\kappa$ B or STAT3, respectively. Cells were seeded in six-well plates and co-transfected with a firefly luciferase reporter and renilla-expressing vectors. After $24 \mathrm{~h}$, luciferase activity was measured using a Dual-Glo ${ }^{\circledR}$ Luciferase Assay System (Promega, Madison, WI, USA). In brief, the cells were lysed in 
lysis buffer containing luciferase substrate for $10 \mathrm{~min}$. Total lysis was achieved with centrifugation at 12,000 rpm for $1 \mathrm{~min}$, and the supernatant was split into three wells in a white 96-well plate to measure firefly luminescence. Dual-Glo ${ }^{\circledR}$ Stop \& Glo ${ }^{\circledR}$ reagent was added to each well. After 10 min, renilla luminescence was measured. The level of firefly luminescence was normalized to renilla luminescence.

\subsection{Immunohistochemistry Staining Analysis}

Paraffin-embedded tumor sections ( $3 \mu \mathrm{m}$ thick) were heated, deparaffinized using xylene, and rehydrated in a graded series of ethanol with a final wash in tap water. Antigen retrieval was performed with target retrieval solution (DAKO) in a decloaking chamber (Biocare Medical, Pacheco, CA, USA). Endogenous peroxidase activity was quenched with hydrogen peroxide. The sections were then incubated with anti-H2AFJ antibody (Sigma-Aldrich, HPA041189, St. Louis, MO, USA) at $4{ }^{\circ} \mathrm{C}$ overnight. A VECTASTAIN ABC peroxidase system (Vector Laboratories, Burlingame, CA, USA) was used to detect the reaction products. Commercially available tissue microarrays were produced by US Biomax Inc. (Rockville, MD, USA) and all human tissues were collected under the Health Insurance Portability and Accountability Act (HIPPA, U.S. Government Publishing Office) approved protocols. Detailed information on all tumor specimens can be found at http://www.biomax.us/index.php. The study was approved by Cardinal Tien Hospital institutional review board approval (CTH-101-3-5-054) in accordance with the Declaration of Helsinki.

\subsection{Statistical Analysis}

SPSS 17.0 software (Informer Technologies, Chicago, IL, USA) was used to analyze statistical significance. One-way ANOVA and Tukey's test were utilized to compare the differential display of H2AFJ gene expression in normal tissues and primary tumors. Pearson's and Spearman tests were performed to estimate the association between H2AFJ levels and the detected parameters. Evaluation of the survival probabilities was determined by Kaplan-Meier analysis and the log-rank test. The Mann-Whitney test was used to analyze the nonparametric data. A $p$-value $<0.05$ in all analyses was considered to be statistically significant.

\section{Conclusions}

This study is the first to document that a histone 2A subfamily member, H2AFJ, has an oncogenic function and is highly expressed in primary tumors of GBM, especially mesenchymal-type GBM, which commonly exhibits a poor TMZ response and unfavorable outcomes in patients. Our results demonstrate that the upregulated expression of $H 2 A F J$ is likely associated with the activation of TNF- $\alpha-N F-\kappa B / I L-6-S T A T 3$ signaling pathways and the recruitment of class I HDAC activity to promote PMT and TMZ resistance in GBM. Fortunately, the finding that HDAC inhibitor tacedinaline, currently a phase II anti-cancer agent, is capable of effectively killing the TMZ-insensitive glioma cells with H2AFJ upregulation provides a feasible strategy for combating the H2AFJ-promoted TMZ resistance in clinical GBM patients.

Supplementary Materials: The following are available online at http://www.mdpi.com/2072-6694/12/1/98/s1, Figure S1: H2AFJ upregulation is detected in mesenchymal-type GBM tissues, Figure S2: The expression of $H 2 A F V, H 2 A F X, H 2 A F Y$ and $H 2 A F Z$ is poorly associated with IDH1 mutation, MGMT promoter methylation and CpG island methylation phenotype (CIMP) in GBM, Figure S3: The expression of $H 2 A F J$ is associated with age, not gender, of low-grade glioma (LGG)/GBM patients, Figure S4: The pharmaceutical inhibition of HDAC3 and NF- $\mathrm{B}$ re-sensitizes the TMZ-insensitive glioma cells with H2AFJ upregulation to TMZ treatment.

Author Contributions: Conception and design, H.-H.L., D.W. and Y.-F.L.; acquisition of data, H.-H.L., C.-H.L., H.-Y.L., C.-H.K., J.-Q.Z. and Y.-H.W.; analysis and interpretation of data, H.-H.L., C.-J.H., F.-P.L., D.W. and Y.-F.L.; writing, review and/or revision of the manuscript, H.-H.L., D.W. and Y.-F.L.; administrative, technical, or material support, L.-S.L., F.-P.L., C.-J.H., D.W. and Y.-F.L.; read and approved the final manuscript, H.-H.L., C.-H.L., H.-Y.L., C.-H.K., J.-Q.Z., Y.-H.W., L.-S.L., F.-P.L., C.-J.H., D.W., Y.-F.L. All authors have read and agreed to the published version of the manuscript. 
Funding: This study was supported by the Ministry of Science and Technology, Taiwan (MOST 108-2320-B-038-017-MY3 to Yuan-Feng Lin and MOST 108-2635-B-038-001 to Hsun-Hua Lee) and by Shuang Ho Hospital, Taipei Medical University (107YSR-03) to Hsun-Hua Lee.

Acknowledgments: The authors would like to thank Michael Hsiao for kindly providing the glioma cell lines and Wen-Yi Ma for her assistance with the in vitro studies.

Conflicts of Interest: The authors declare no conflict of interest.

\section{References}

1. Louis, D.N.; Perry, A.; Reifenberger, G.; von, D.A.; Figarella-Branger, D.; Cavenee, W.K.; Ohgaki, H.; Wiestler, O.D.; Kleihues, P.; Ellison, D.W. The 2016 World Health Organization Classification of Tumors of the Central Nervous System: A summary. Acta Neuropathol. 2016, 131, 803-820. [CrossRef] [PubMed]

2. Chandana, S.R.; Movva, S.; Arora, M.; Singh, T. Primary brain tumors in adults. Am. Fam. Physician 2008, 77, 1423-1430. [PubMed]

3. Urbanska, K.; Sokolowska, J.; Szmidt, M.; Sysa, P. Glioblastoma multiforme-An overview. Contemp. Oncol. (Pozn.) 2014, 18, 307-312. [PubMed]

4. Ohka, F.; Natsume, A.; Wakabayashi, T. Current trends in targeted therapies for glioblastoma multiforme. Neurol. Res. Int. 2012, 2012, 878425. [CrossRef]

5. Batash, R.; Asna, N.; Schaffer, P.; Francis, N.; Schaffer, M. Glioblastoma Multiforme, Diagnosis and Treatment; Recent Literature Review. Curr. Med. Chem. 2017, 24, 3002-3009. [CrossRef]

6. Verhaak, R.G.; Hoadley, K.A.; Purdom, E.; Wang, V.; Qi, Y.; Wilkerson, M.D.; Miller, C.R.; Ding, L.; Golub, T.; Mesirov, J.P.; et al. Integrated genomic analysis identifies clinically relevant subtypes of glioblastoma characterized by abnormalities in PDGFRA, IDH1, EGFR, and NF1. Cancer Cell 2010, 17, 98-110. [CrossRef]

7. Zhang, C.; Moore, L.M.; Li, X.; Yung, W.K.; Zhang, W. IDH1/2 mutations target a key hallmark of cancer by deregulating cellular metabolism in glioma. Neuro Oncol. 2013, 15, 1114-1126. [CrossRef]

8. Labussiere, M.; Idbaih, A.; Wang, X.W.; Marie, Y.; Boisselier, B.; Falet, C.; Paris, S.; Laffaire, J.; Carpentier, C.; Criniere, E.; et al. All the 1p19q codeleted gliomas are mutated on IDH1 or IDH2. Neurology 2010, 74, 1886-1890. [CrossRef]

9. Dubbink, H.J.; Taal, W.; Van Marion, R.; Kros, J.M.; Van Heuvel, I.; Bromberg, J.E.; Zonnenberg, B.A.; Zonnenberg, C.; Postma, T.J.; Gijtenbeek, J.M.; et al. IDH1 mutations in low-grade astrocytomas predict survival but not response to temozolomide. Neurol 2009, 73, 1792-1795. [CrossRef]

10. Minniti, G.; Scaringi, C.; Arcella, A.; Lanzetta, G.; Di, S.D.; Scarpino, S.; Bozzao, A.; Pace, A.; Villani, V.; Salvati, M.; et al. IDH1 mutation and MGMT methylation status predict survival in patients with anaplastic astrocytoma treated with temozolomide-based chemoradiotherapy. J. Neurooncol. 2014, 118, 377-383. [CrossRef]

11. Brandes, A.A.; Franceschi, E.; Tosoni, A.; Blatt, V.; Pession, A.; Tallini, G.; Bertorelle, R.; Bartolini, S.; Calbucci, F.; Andreoli, A.; et al. MGMT promoter methylation status can predict the incidence and outcome of pseudoprogression after concomitant radiochemotherapy in newly diagnosed glioblastoma patients. J. Clin. Oncol. 2008, 26, 2192-2197. [CrossRef] [PubMed]

12. Li, H.; Li, J.; Cheng, G.; Zhang, J.; Li, X. IDH mutation and MGMT promoter methylation are associated with the pseudoprogression and improved prognosis of glioblastoma multiforme patients who have undergone concurrent and adjuvant temozolomide-based chemoradiotherapy. Clin. Neurol. Neurosurg. 2016, 151, 31-36. [CrossRef] [PubMed]

13. Lee, A.; Malakhov, N.; Sheth, N.; Wang, A.; Han, P.; Schreiber, D. Patterns of care and outcomes of chemoradiation versus radiation alone for MGMT promoter unmethylated glioblastoma. Clin. Neurol. Neurosurg. 2018, 170, 127-131. [CrossRef] [PubMed]

14. Alabert, C.; Jasencakova, Z.; Groth, A. Chromatin Replication and Histone Dynamics. Adv. Exp. Med. Biol. 2017, 1042, 311-333. [PubMed]

15. Testa, E.; Nardozi, D.; Antinozzi, C.; Faieta, M.; Di Cecca, S.; Caggiano, C.; Fukuda, T.; Bonanno, E.; Zhenkun, L.; Maldonado, A.; et al. H2AFX and MDC1 promote maintenance of genomic integrity in male germ cells. J. Cell Sci. 2018, 131, jcs214411. [CrossRef] 
16. Monteiro, F.L.; Vitorino, R.; Wang, J.; Cardoso, H.; Laranjeira, H.; Simoes, J.; Caldas, M.; Henrique, R.; Amado, F.; Williams, C.; et al. The histone H2A isoform Hist2h2ac is a novel regulator of proliferation and epithelial-mesenchymal transition in mammary epithelial and in breast cancer cells. Cancer Lett. 2017, 396, 42-52. [CrossRef]

17. Fan, W.; Zhou, K.; Zhao, Y.; Wu, W.; Chen, H.; Jin, L.; Chen, G.; Shi, J.; Wei, Q.; Zhang, T.; et al. Possible association between genetic variants in the H2AFX promoter region and risk of adult glioma in a Chinese Han population. J. Neurooncol. 2011, 105, 211-218. [CrossRef]

18. Juarez-Velazquez, R.; Reyes-Leon, A.; Salas-Labadia, C.; Rivera-Luna, R.; Velasco-Hidalgo, L.; Lopez-Hernandez, G.; Lopez-Santiago, N.; Paredes-Aguilera, R.; Dominguez-Lopez, A.; Bernaldez, R.; et al. Significance of CASP8AP2 and H2AFZ expression in survival and risk of relapse in children with acute lymphoblastic leukemia. Leuk. Lymphoma 2014, 55, 2305-2311. [CrossRef]

19. Jin, X.M.; Kim, H.N.; Shin, M.H.; Lee, I.K.; Lee, J.S.; Lee, J.H.; Kim, H.J.; Choi, J.S.; Juhng, S.W.; Choi, C. $\mathrm{H} 2 \mathrm{AFX}$ polymorphisms are associated with decreased risk of diffuse large B cell lymphoma in Koreans. DNA Cell Biol. 2011, 30, 1039-1044. [CrossRef]

20. Segerman, A.; Niklasson, M.; Haglund, C.; Bergstrom, T.; Jarvius, M.; Xie, Y.; Westermark, A.; Sonmez, D.; Hermansson, A.; Kastemar, M.; et al. Clonal Variation in Drug and Radiation Response among Glioma-Initiating Cells Is Linked to Proneural-Mesenchymal Transition. Cell Rep. 2016, 17, 2994-3009. [CrossRef]

21. Bhat, K.P.L.; Balasubramaniyan, V.; Vaillant, B.; Ezhilarasan, R.; Hummelink, K.; Hollingsworth, F.; Wani, K.; Heathcock, L.; James, J.D.; Goodman, L.D.; et al. Mesenchymal differentiation mediated by NF-kappaB promotes radiation resistance in glioblastoma. Cancer Cell 2013, 24, 331-346. [CrossRef] [PubMed]

22. Richards, D.A.; Boehm, K.A.; Waterhouse, D.M.; Wagener, D.J.; Krishnamurthi, S.S.; Rosemurgy, A.; Grove, W.; Macdonald, K.; Gulyas, S.; Clark, M.; et al. Gemcitabine plus CI-994 offers no advantage over gemcitabine alone in the treatment of patients with advanced pancreatic cancer: Results of a phase II randomized, double-blind, placebo-controlled, multicenter study. Ann. Oncol. 2006, 17, 1096-1102. [CrossRef] [PubMed]

23. Aguirre-Gamboa, R.; Gomez-Rueda, H.; Martinez-Ledesma, E.; Martinez-Torteya, A.; Chacolla-Huaringa, R.; Rodriguez-Barrientos, A.; Tamez-Pena, J.G.; Trevino, V. SurvExpress: An online biomarker validation tool and database for cancer gene expression data using survival analysis. PLoS ONE 2013, 8, e74250. [CrossRef] [PubMed]

24. Phillips, H.S.; Kharbanda, S.; Chen, R.; Forrest, W.F.; Soriano, R.H.; Wu, T.D.; Misra, A.; Nigro, J.M.; Colman, H.; Soroceanu, L.; et al. Molecular subclasses of high-grade glioma predict prognosis, delineate a pattern of disease progression, and resemble stages in neurogenesis. Cancer Cell 2006, 9, 157-173. [CrossRef]

25. Gao, Y.F.; Mao, X.Y.; Zhu, T.; Mao, C.X.; Liu, Z.X.; Wang, Z.B.; Li, L.; Li, X.; Yin, J.Y.; Zhang, W.; et al. COL3A1 and SNAP91: Novel glioblastoma markers with diagnostic and prognostic value. Oncotarget 2016, 7, 70494-70503. [CrossRef]

26. Zhang, X.; Sun, S.; Pu, J.K.; Tsang, A.C.; Lee, D.; Man, V.O.; Lui, W.M.; Wong, S.T.; Leung, G.K. Long non-coding RNA expression profiles predict clinical phenotypes in glioma. Neurobiol. Dis. 2012, 48, 1-8. [CrossRef]

27. Rees, M.G.; Seashore-Ludlow, B.; Cheah, J.H.; Adams, D.J.; Price, E.V.; Gill, S.; Javaid, S.; Coletti, M.E.; Jones, V.L.; Bodycombe, N.E.; et al. Correlating chemical sensitivity and basal gene expression reveals mechanism of action. Nat. Chem. Biol. 2016, 12, 109-116. [CrossRef]

28. Seashore-Ludlow, B.; Rees, M.G.; Cheah, J.H.; Cokol, M.; Price, E.V.; Coletti, M.E.; Jones, V.; Bodycombe, N.E.; Soule, C.K.; Gould, J.; et al. Harnessing Connectivity in a Large-Scale Small-Molecule Sensitivity Dataset. Cancer Discov. 2015, 5, 1210-1223. [CrossRef]

29. Olmez, I.; Love, S.; Xiao, A.; Manigat, L.; Randolph, P.; McKenna, B.D.; Neal, B.P.; Boroda, S.; Li, M.; Brenneman, B.; et al. Targeting the mesenchymal subtype in glioblastoma and other cancers via inhibition of diacylglycerol kinase alpha. Neuro Oncol. 2018, 20, 192-202. [CrossRef]

30. Hegi, M.E.; Diserens, A.C.; Gorlia, T.; Hamou, M.F.; de, T.N.; Weller, M.; Kros, J.M.; Hainfellner, J.A.; Mason, W.; Mariani, L.; et al. MGMT gene silencing and benefit from temozolomide in glioblastoma. N. Engl. J. Med. 2005, 352, 997-1003. [CrossRef]

31. Yu, Z.; Chen, Y.; Wang, S.; Li, P.; Zhou, G.; Yuan, Y. Inhibition of NF-kappaB results in anti-glioma activity and reduces temozolomide-induced chemoresistance by down-regulating MGMT gene expression. Cancer Lett. 2018, 428, 77-89. [CrossRef] [PubMed] 
32. Brassesco, M.S.; Roberto, G.M.; Morales, A.G.; Oliveira, J.C.; Delsin, L.E.; Pezuk, J.A.; Valera, E.T.; Carlotti, C.G., Jr.; Rego, E.M.; de Oliveira, H.F.; et al. Inhibition of NF-kappa B by Dehydroxymethylepoxyquinomicin Suppresses Invasion and Synergistically Potentiates Temozolomide and gamma -Radiation Cytotoxicity in Glioblastoma Cells. Chemother. Res. Pract. 2013, 2013, 593020. [PubMed]

33. Bredel, M.; Bredel, C.; Juric, D.; Duran, G.E.; Yu, R.X.; Harsh, G.R.; Vogel, H.; Recht, L.D.; Scheck, A.C.; Sikic, B.I. Tumor necrosis factor-alpha-induced protein 3 as a putative regulator of nuclear factor-kappaB-mediated resistance to O6-alkylating agents in human glioblastomas. J. Clin. Oncol. 2006, 24, 274-287. [CrossRef] [PubMed]

34. Carro, M.S.; Lim, W.K.; Alvarez, M.J.; Bollo, R.J.; Zhao, X.; Snyder, E.Y.; Sulman, E.P.; Anne, S.L.; Doetsch, F.; Colman, H.; et al. The transcriptional network for mesenchymal transformation of brain tumours. Nature 2010, 463, 318-325. [CrossRef] [PubMed]

35. Rolhion, C.; Penault-Llorca, F.; Kemeny, J.L.; Lemaire, J.J.; Jullien, C.; Labit-Bouvier, C.; Finat-Duclos, F.; Verrelle, P. Interleukin-6 overexpression as a marker of malignancy in human gliomas. J. Neurosurg. 2001, 94, 97-101. [CrossRef] [PubMed]

36. Vlachostergios, P.J.; Hatzidaki, E.; Papandreou, C.N. MGMT repletion after treatment of glioblastoma cells with temozolomide and O6-benzylguanine implicates NFkappaB and mutant p53. Neurol. Res. 2013, 35, 879-882. [CrossRef]

37. Bredel, M.; Scholtens, D.M.; Yadav, A.K.; Alvarez, A.A.; Renfrow, J.J.; Chandler, J.P.; Yu, I.L.; Carro, M.S.; Dai, F.; Tagge, M.J.; et al. NFKBIA deletion in glioblastomas. N. Engl. J. Med. 2011, 364, 627-637. [CrossRef]

38. Song, N.; Liu, J.; An, S.; Nishino, T.; Hishikawa, Y.; Koji, T. Immunohistochemical Analysis of Histone H3 Modifications in Germ Cells during Mouse Spermatogenesis. Acta Histochem. Cytochem. 2011, 44, $183-190$. [CrossRef]

39. Lawrence, M.; Daujat, S.; Schneider, R. Lateral Thinking: How Histone Modifications Regulate Gene Expression. Trends Genet. 2016, 32, 42-56. [CrossRef]

40. McCabe, M.T.; Mohammad, H.P.; Barbash, O.; Kruger, R.G. Targeting Histone Methylation in Cancer. Cancer J. 2017, 23, 292-301. [CrossRef]

41. Michalak, E.M.; Burr, M.L.; Bannister, A.J.; Dawson, M.A. The roles of DNA, RNA and histone methylation in ageing and cancer. Nat. Rev. Mol. Cell Biol. 2019, 20, 573-589. [CrossRef] [PubMed]

42. Waterborg, J.H. Evolution of histone H3: Emergence of variants and conservation of post-translational modification sites. Biochem. Cell Biol. 2012, 90, 79-95. [CrossRef] [PubMed]

43. Corujo, D.; Buschbeck, M. Post-Translational Modifications of H2A Histone Variants and Their Role in Cancer. Cancers 2018, 10, 59. [CrossRef] [PubMed]

44. Barthel, F.P.; Johnson, K.C.; Varn, F.S.; Moskalik, A.D.; Tanner, G.; Kocakavuk, E.; Anderson, K.J.; Abiola, O.; Aldape, K.; Alfaro, K.D.; et al. Longitudinal molecular trajectories of diffuse glioma in adults. Nature 2019, 576, 112-120. [CrossRef] [PubMed]

(C) 2019 by the authors. Licensee MDPI, Basel, Switzerland. This article is an open access article distributed under the terms and conditions of the Creative Commons Attribution (CC BY) license (http://creativecommons.org/licenses/by/4.0/). 